\title{
Pulsation reduction system for positive displacement pumps
}

\author{
T Hendriks MHWirth GmbH, Germany
}

R Jansen MHWirth GmbH, Germany

\begin{abstract} reliability of the piston diaphragm pumps leads to a more stable process. the entire system. diaphragm pumps.

Brief description of functionality: throttle effects. This effect is similar to a shock absorber. following benefits by using it in a tailings and storage facility:

- Elimination of pressure surges and water hammer effects.

- Minimum vibration at highest stroke rates.

- Cost-value ratio.

- Retrofitting of existing pumps.
\end{abstract}

Positive displacement pumps are a crucial part of the entire process of a tailings and storage facility. Any pump downtime or unplanned maintenance directly affects the productivity. Therefore, increasing the

When operating piston diaphragm pumps, the oscillating movement of the pump pistons results in asymmetrical flows, both in the suction side and at the discharge side of the pump. These asymmetrical flow rates lead to pressure pulsations and to vibrations in the pump itself and the adjacent piping system, having a negative impact on the pump operation. In the suction side of the pump, these pulsations can cause cavitation that reduces the pump reliability and poses a risk for damages, not only for the pump, but also for

The paper will present a new pulsation reduction system (PRS) invented by MHWirth. This PRS is - in contrast to conventional pulsation dampers-suitable to eliminate high-frequency pulsation of reciprocating

The new damper is connected to the propelling fluid chamber of the diaphragms. The propelling fluid, a hydraulic oil, is used as a damping medium, converting the pulsation energy described above into heat by

The paper will also explain the PRS effects, present operational data from field application and address the

Keywords: piston diaphragm pumps, paste pumping, tailings storage facility, pulsation, cavitation, pressure surge

\section{Introduction}

Positive displacement pumps (PD pumps) have been used in various applications such as slurry pipeline transfer, mine de-watering, hydraulic ore hoisting, autoclave feed, and digester feed for many years. The operational characteristics and the effects to consider have recently been presented at the ALTA Conference in Perth in 2017 (Jolly \& Cocks 2017) and the topic described in this document expands upon that work. In the remainder of this paper, we focus on PD pumps used in tailings transfer operations.

The pumps are a triplex single or duplex double acting design and are preferably used in projects where traditional centrifugal pumps reached their discharge pressure limitation. An electric motor driven crank mechanism is connected via connecting rods and crossheads to the pistons. With each stroke, hydraulic oil is pumped into housings which are equipped with a flexible diaphragm. The diaphragm separates and protects the hydraulic oil and the moving parts behind it from the slurry which is in front of the diaphragm. 
During the discharge stroke of the piston, hydraulic oil will move the flexible diaphragm forward, and slurry which is present at the other side of the diaphragm is discharged through the discharge non-return valve. When the piston moves backward, the suction pressure on the slurry side of the diaphragm will push the diaphragm backwards, and suction pressure will fill the diaphragm housing with slurry through the non-return suction valve. The referenced slurry pump model used for this paper is an MHWirth triplex pump model TPM 2200-20. Figure 1 shows a partial sectional view of the operating principle of this piston diaphragm pump.

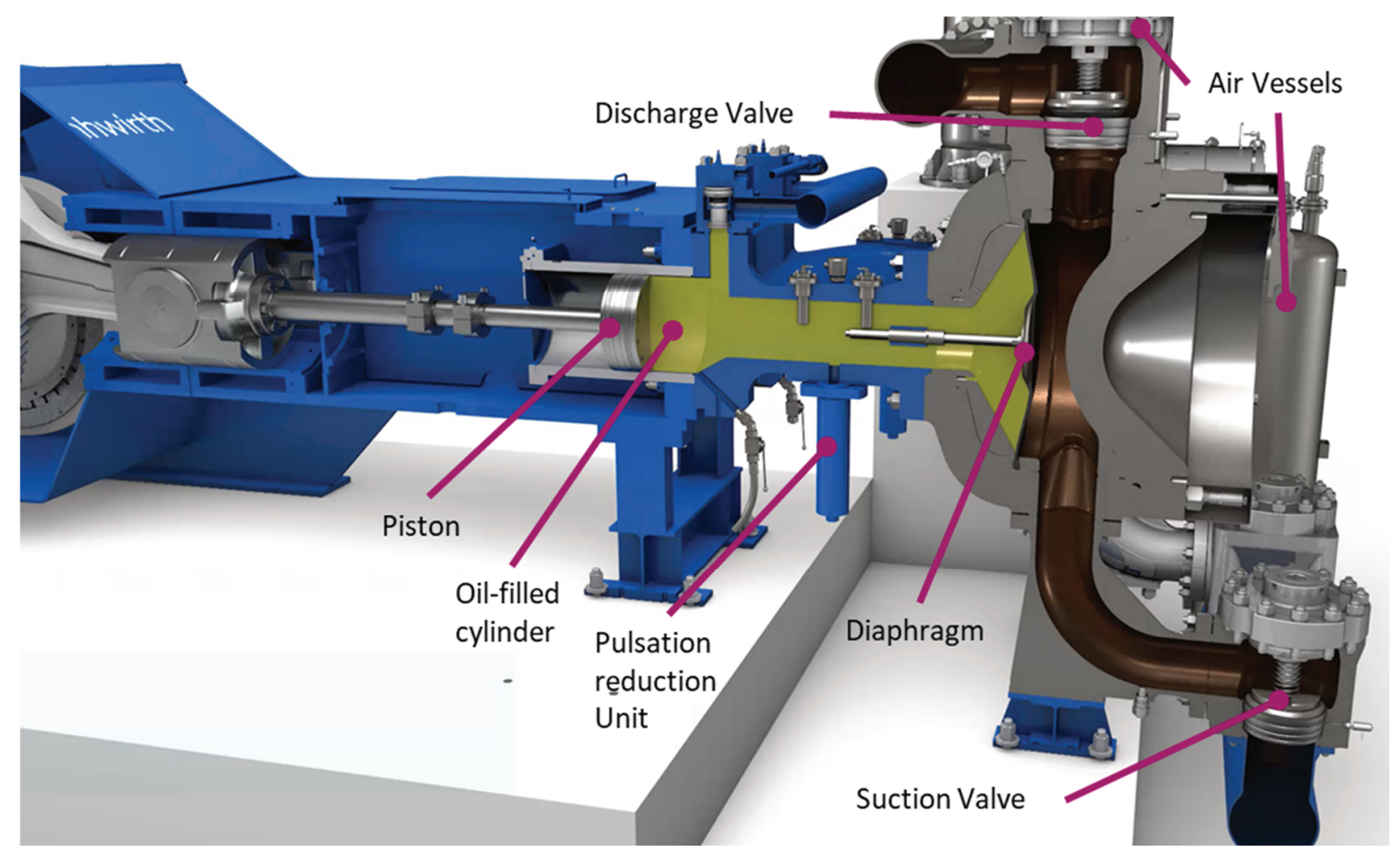

\section{Figure 1 Sectional view of a triplex diaphragm pump's fluid end}

One effect which needs to be considered while operating positive displacement pumps is pulsation. Rotating parts, oscillating movement, suction and discharge of slurry, acceleration and velocities are mutually dependent on each other and cause pulsations (Vlot 2018).

A difference is found between two types of pulsations. The first is a low-frequency, flow-induced, pulsation where, due to the reciprocating movement of the triplex pump, each of the three strokes discharges at a different angel of the crankshaft resulting in a maximum variation of the pump flow of $23 \%$. Due to the inertia of the accelerated slurry, this fluctuation of the flow causes pressure pulsations, the frequency of which depends on the speed of the pump. In a triplex pump, the frequency of the fundamental oscillation of this pulsation is three times the speed of the pump. If, for example, the crankshaft of a triplex pump rotates at a speed of $60 \mathrm{rpm}$ or $1 \mathrm{~Hz}$, this results in a fundamental oscillation of the flow-induced pulsation of $3 \mathrm{~Hz}$. In addition to this fundamental oscillation, there are harmonics, the oscillation frequency of which is an integral multiple of the fundamental frequency.

This pulsation is reduced by the air vessel installed in the pump discharge line to lower the pressure peaks down to $<3 \%$ residual peak-to-peak pulsation. These effects also occur in the suction line of the pump and are considered by the net positive suction head (NPSH) calculation when designing the pump.

The second types of pulsations on which this publication will focus are much higher frequency pulsations. These occur during each suction and discharge stroke while pumping. The frequencies of these pulsations are usually in the range of 15 to $50 \mathrm{~Hz}$ at a pressure amplitude of approximately 10 bar and above. Especially during the suction stroke, these pulsations can be a source of multiple cavitation events (Schade 2009). 
Whereas the pulsations during the discharge stroke can cause significant mechanical vibrations in the discharge line which may harm the pump and other process components connected to the discharge line.

In this paper we will describe the origin and the effect of high-frequency pulsations caused by a triplex pump. Based on field measurements the initial situation will be described and after setting the objective we will outline the functionality and scope of the pulsation reduction system (PRS) followed by a critical assessment.

\section{$2 \quad$ High-frequency fluid pressure pulsation}

Comparable to mechanical or electrical systems, hydraulic systems are subject to oscillation effects in a similar manner. In order to describe and calculate hydraulic pulsation effects, similar parameters and formulas known from electrical resonant circuits, also known as inductor-capacitor circuits, are used.

The background of the hydraulic oscillation is a relation between potential and kinetic energy in the hydraulic system (Murrenhoff 1998).

A fluid in a pipeline is always mass-adherent and follows the laws of inertia. In order to describe the inertia of a fluid column, the term 'hydraulic inductance' $L_{H}$ is introduced:

$$
L_{H}=\frac{\Delta p}{\dot{Q}}
$$

where $\Delta p$ represents the pressure difference to allow a change in the flow $\dot{Q}$.

The hydraulic inductance describes the resistance of a hydraulic fluid to acceleration effect. It is comparable to electrical inductance, which describes the behaviour of electric coils in oscillating circuits. Typical examples of pronounced hydraulic inductances are high-density fluids in long pipelines. For such a case, the inductance of a fluid column with a fluid density of $\rho$ in a pipe with the length I and a cross-section area $A$ can be calculated as follows:

$$
L_{H}=\frac{l \rho}{A}
$$

In addition to the inductance, an electrical resonant circuit also requires a capacitance. Also in hydraulics, there is the concept of capacitance. The 'hydraulic capacitance' $C_{H}$ describes the ratio of flow $Q$ to pressure change $\dot{p}$ :

$$
C_{H}=\frac{Q}{\dot{p}}
$$

Typical examples of hydraulic capacitances are large pressurised volumes filled with a compressible fluid. If the compression modulus $E_{F L}$ of a liquid and its volume $V_{0}$ is known, the hydraulic capacitance can also be defined as:

$$
C_{H}=\frac{V_{0}}{E_{F L}}
$$

For a hydraulic volume with compressible fluid, the hydraulic capacitance $C_{H}$ can also be expressed as the quotient of displaced volume $\Delta V$ and the resultant pressure change $\Delta p$.

$$
C_{H}=\frac{\Delta V}{\Delta p}
$$

The natural frequency $f_{0}$ of a hydraulic system, consisting of an inductance and a capacitance, is defined in analogy to the natural frequency of an electric resonant circuit to:

$$
f_{0}=\frac{1}{2 \pi \sqrt{L_{H} C_{H}}}
$$

An example for an oscillating fluid system in a triplex pump is shown in Figure 2. In this case, it consists of two hydraulic capacitances $C_{H 1}$ and $C_{H 2}$, and a hydraulic inductance $L_{H 1}$ which connects the two capacitances. In this configuration, the $C_{H 1}$ capacitance is made up of the slurry and propelling fluid volume of a diaphragm housing, while the $C_{\mathrm{H} 2}$ capacitance represents the discharge air vessel. Both capacitances can store potential energy in the form of pressure energy, whereas kinetic energy prevails in the pipeline when there is pressure 
equalisation between the two capacitances. The diaphragm housing $C_{H 1}$ can absorb a certain amount of fluid volume due to the flexibility of its housing wall and the compressibility of the fluid, even if the housing is already completely filled with fluid. The quotient of the absorbed fluid volume and the resulting pressure increase is the value of the hydraulic capacitance $C_{H 1}$, as already shown in the above formula. If the air vessel takes up the same amount of fluid, this leads to a much smaller pressure increase compared to that in the diaphragm housing, since the large air volume in the air vessel, usually several hundred litres, leads to hardly any noticeable pressure increase. This means that the hydraulic capacitance of the air vessel is several times higher than that of the diaphragm housing. For this reason, the air vessel can be regarded as a constant pressure source. It does not play a role in the calculation of the natural frequency of the hydraulic oscillation. Therefore, the natural frequency $f_{0}$ depends only on the capacitance of the diaphragm housing $C_{H 1}$ and the inductance of the connecting line $L_{H}$.

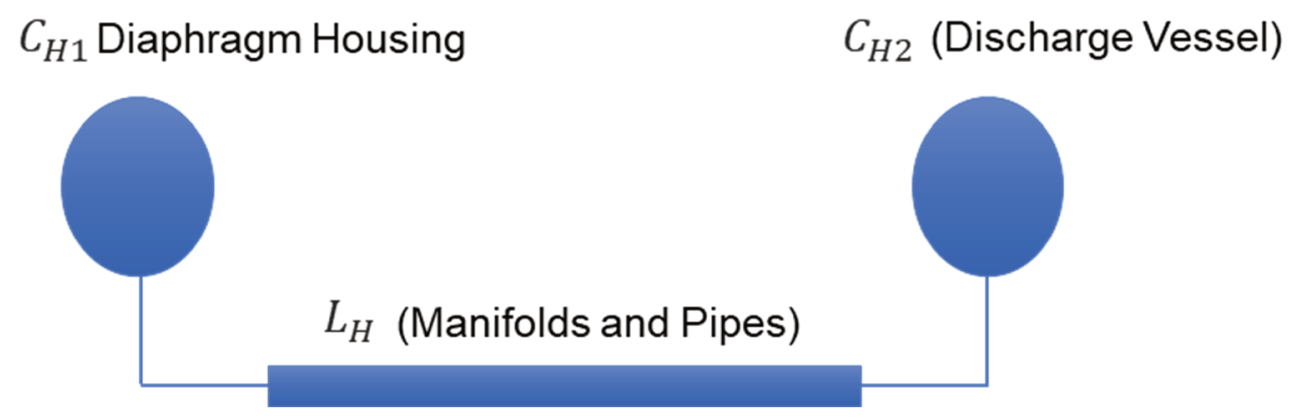

Figure 2 Example of an oscillating fluid system in a triplex pump

$$
f_{0}=\frac{1}{2 \pi \sqrt{L_{H} C_{H 1}}}
$$

For a positive displacement slurry pump, on which the measurements shown in Figure 3 have been carried out, the following values are obtained for the hydraulic capacitances and inductances between the outer diaphragm housing and the discharge air vessel. If the pump is operated with water as medium, the hydraulic inductance is calculated for the pipes between the diaphragm housing and the air vessel.

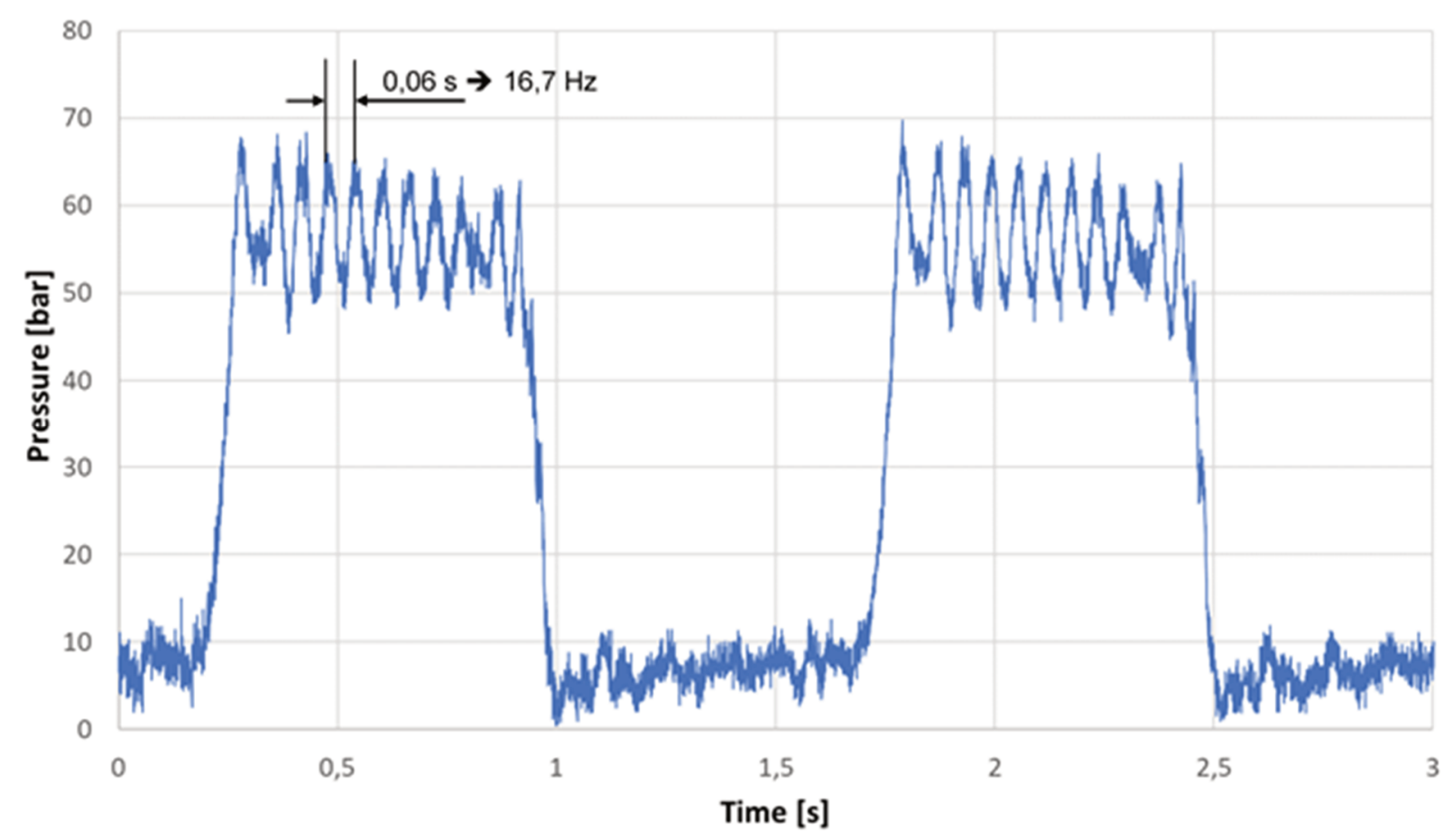

Figure 3 Measurement of the chamber pressure during an in-house test 


$$
L_{H}=\frac{l \rho}{A}=\frac{11.9 m * 1,000 \frac{\mathrm{kg}}{\mathrm{m}^{3}}}{\pi * 0.25 *(0.254 \mathrm{~m})^{2}}=234,850 \frac{\mathrm{kg}}{\mathrm{m}^{4}}
$$

By means of pressure measurements and simulations, the pressure increase curve and thus the hydraulic capacitance of the diaphragm housing could be estimated as follows:

$$
C_{H 1}=\frac{\Delta V}{\Delta p}=\frac{0.00101 \mathrm{~m}^{3}}{3 * 10^{6} \frac{\mathrm{N}}{\mathrm{m}^{2}}}=0.367 * 10^{-9} \frac{\mathrm{m}^{5}}{\mathrm{~N}}
$$

With these values, the hydraulic oscillation between the diaphragm housing and the air vessel results in a frequency of:

$$
f_{0}=\frac{1}{2 \pi \sqrt{L_{H} C_{H 1}}}=\frac{1}{2 \pi \sqrt{234,850 \frac{\mathrm{kg}}{\mathrm{m}^{4}} * 0.367 * 10^{-9} \frac{\mathrm{m}^{5}}{N}}}=17.1 \mathrm{~Hz}
$$

It should be mentioned that for various reasons there are differences in the calculated frequencies of measured pressure pulsations. On one hand, the triplex pump has several oscillation sub-systems, which partly influence each other. Since there are three diaphragm housings, which all have different line lengths up to the air vessel, there are three different natural frequencies here alone. There are temporary connections between these oscillating circuits, if, for example, two piston chambers convey simultaneously into the outlet manifold via open non-return valves. This can also lead to further forms of oscillation between these diaphragm chambers. On the other hand, a distinction between the main inductances and capacitances in the pump is not always clear. So naturally the pipe also has a capacitance, as potential energy can be stored in the pipe itself due to its large cross-sections. In addition, there are other installations in the pipeline system, such as valves, extensions, bends, and dead water areas, which make it difficult to determine the inductances.

The suction vessel is located on the suction side of the pump. Here too, oscillating hydraulic systems are generated between the suction air vessel and the individual diaphragm chambers. If cavitation effects occur during the suction process, the free oscillation can often be interrupted by this cavitation, which is why the oscillations in this case often do not correspond to a normal sinusoidal oscillation.

\subsection{Effect on pump performance and process}

The high-frequency pulsations are present in every reciprocating, positive displacement pump system and can be measured using a pump monitoring system like the MHWirth WPMS ${ }^{\mathrm{TM}}$ which allows monitoring of each cylinder in real time. Figure 3 shows the pressure inside a diaphragm housing of a triplex pump running at $40 \mathrm{rpm}$. During this measurement, the pump was operated with water as medium, and the charging pressure was set to $6.5 \mathrm{bar}$, and the discharge pressure to $57 \mathrm{bar}$.

Figure 3 shows the pressure curve for two complete pump cycles. As can be seen, especially during the discharge phase, there are pressure pulsations with a peak-to-peak amplitude of about 16 bar. The period time of this oscillation is $0.06 \mathrm{~s}$ which results in a frequency of $16.7 \mathrm{~Hz}$. This corresponds very well with the theoretical considerations made at the previous chapter $(17.1 \mathrm{~Hz})$. Pulsations also occur during the suction phase, but here they are not as pronounced in terms of amplitude.

This high-frequency pulsation can lead to cavitation and water hammer effects, similar to the so-called Zhukovsky pressure surges. In extreme cases, these events can have a damaging effect on the pump and could lead to material fatigue in the adjacent piping system, especially when they get in resonance with the pulsation. Often these pulsations also limit the maximum speed of the pump, since the height of the pulsation increases over-proportionally with the pump speed. 


\subsection{Objective}

The field measurements above and the description of the negative effects of the high-frequency pulsations within the pump system raise the question of how to deal with that phenomena. Basically, there are four options available:

1. Ignore and operate as usual.

2. Consider the effects in the pump design, e.g. component size, thickness, nominal speed.

3. Avoid its origin.

4. Eliminate the high-frequency pulsation.

The first option is no choice, as the effects described have a negative influence on pump performance and reliability which cannot be disregarded.

If considered for in the design, it has to be checked how this correlates with the pump performance and the cost involved. For example, to avoid the risk for possible cavitation damage, the pre-charge pressure could be increased to keep it above the slurry vapour pressure at all times and temperatures, but this will increase the cost on the operator side.

As the high-frequency pulsations are inherently given in such a pumping system, it cannot be avoided. At the design stage, an attempt can be made to minimise the pulsation energy, but certain criteria must be met at the design phase, which cannot easily be disregarded. In the case of the PD pump, for example, the sizing of the components predicts the capacitance and inductance.

In addition, an attempt can be made to change the frequency of the pulsation in order to minimise possible resonance effects on adjacent systems. However, even here the possibilities are limited, and it is often difficult to find out the relevant resonance frequencies in the adjacent piping system in the design phase of the pump.

Thus, the last option is to eliminate them as soon as they occur. The objective is therefore to develop a system which is able to reduce the high-frequency pulsations to a maximum possible.

\section{Pulsation reduction system}

Conventional hydraulic dampers such as air vessels or bladder accumulators cannot eliminate the high-frequency pressure pulsations shown here, as these are designed to the flow-induced pulsations. To the contrary, these dampers are even part of the high-frequency oscillation system, as shown in the theoretical considerations on the origin of the high-frequency pulsation. The purpose of these conventional types of dampers is to de-couple the pump from the rest of the piping system, as the gas filling of the dampers acts like a soft spring that compensates for the uneven flow of the individual pistons. If there is a short-term increase in the flow, the gas-filled damper can easily absorb the additional volume. This compresses the gas in the damper, and the high compressibility of the gas only leads to a marginal increase in pressure. If the pump flow is reduced below the average flow of the pump, the previously absorbed volume can be emitted from the damper, causing the gas to expand again. These effects have the consequence that the flow downstream of the pressure damper and on the suction side upstream of the suction damper is smoothed and the pressure peaks are reduced.

The function of these gas-filled dampers can best be compared to the suspension in a car driving on an uneven road. Each pothole on the road represents a peak flow rate which is generated by the alternating piston movement. When the car drives through a pothole, the spring in the car's suspension reduces the mechanical impact on the passenger cabin, just as gas-filled dampers reduce the pressure peak that occurs when the pump produces a peak in the flow. The frequency of the shocks depends on the distribution of the potholes on the road and the speed of the vehicle, just as the frequency of the flow-induced pulsation in the pump depends on the number of pistons and the speed of the pump. 
In the case of a vehicle, the uneven road causes yet another vibration that does not depend on the speed of the vehicle or the frequency of the potholes. This oscillation is determined by the natural frequency of the vehicle with its suspension. The springs of the chassis store energy. Like a spring pendulum, this energy is converted into a vertical movement of the passenger cell and then stored again as potential energy in the springs. In a car, this natural oscillation is eliminated by shock absorbers arranged parallel to the springs in the suspension. The shock absorbers convert the oscillation energy into heat, so that the vertical oscillation of the vehicle quickly subsides after a pothole.

Up to now, there has been no such shock absorber in a slurry pump that converts pulsation energy to heat. Although it would be possible to insert a throttle orifice plate into the pipeline between the diaphragm housing and the air vessel in order to reduce the pulsation, this would inadmissibly throttle the high flow rate of the pump and reduce the efficiency of the pump immensely. On the suction side of the pump, such an orifice would have a negative effect on the necessary NPSH value, since the tendency to cavitation would be considerably increased.

For this reason, MHWirth developed the patented pulsation reduction system (PRS), which eliminates the high-frequency pulsations without noticeably affecting the efficiency of the pump. A first application is running reliably at a customer site since January 2019. The four pumps installed are MHWirth duplex double acting model DPM1250-22 each providing $715 \mathrm{~m}^{3} / \mathrm{h}$ at 45 bar. In Figure 4 you can see pressure measurements of this application performed on a duplex pump with and without PRS installed to the pump.
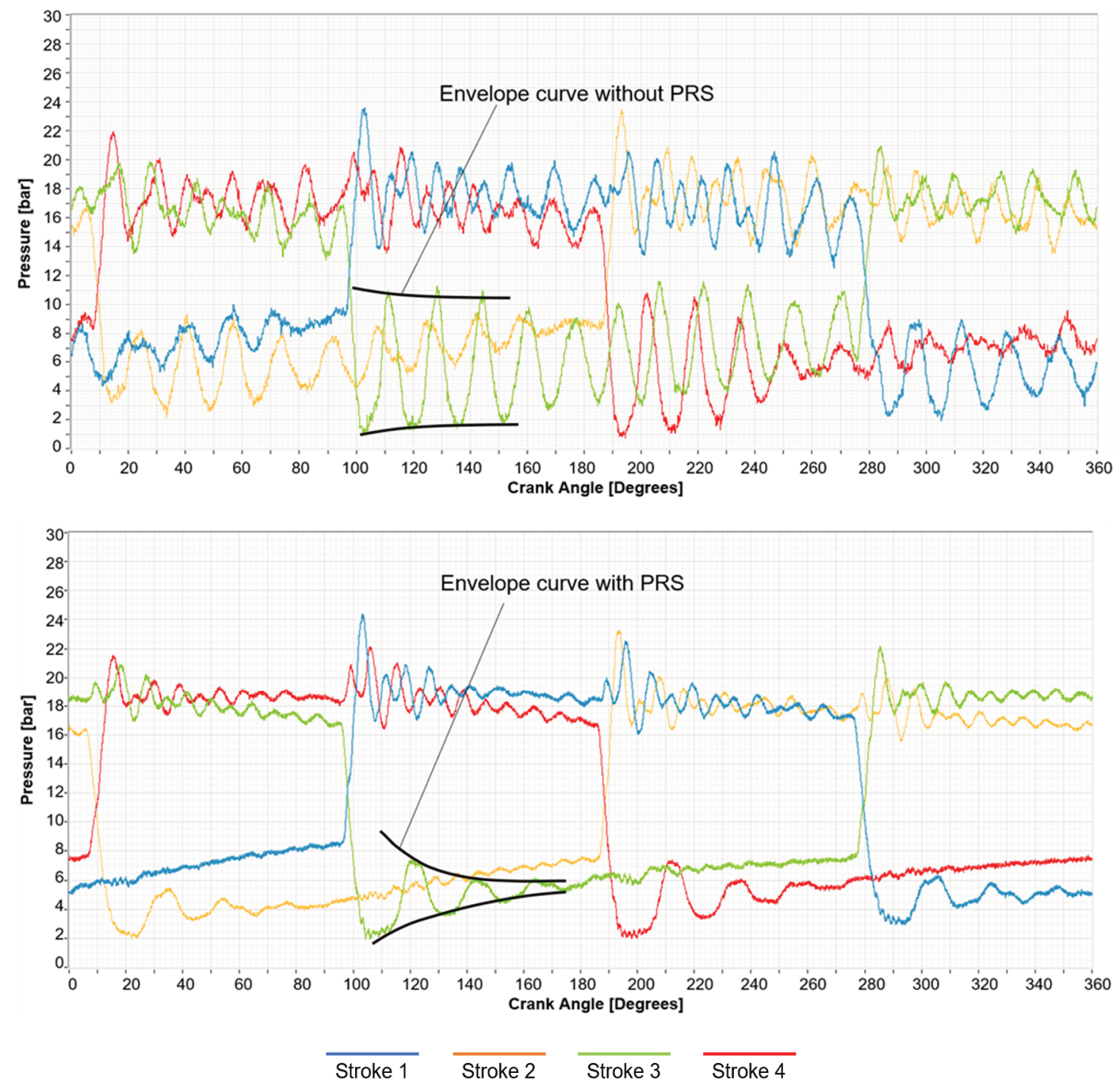

Figure 4 Chamber pressures of a duplex diaphragm pump plotted over the crank angle without PRS (top) and with PRS (bottom) 
In the upper part of Figure 4, the pressures in the four diaphragm chambers of the pump can be seen during a complete rotation of the crankshaft. The suction pressure of the pump here is about 6 bar and the discharge pressure about 19 bar. As can be seen, all chamber pressures are superimposed by the high-frequency pressure pulsations already described, both during the suction and discharge phases. The natural friction in the system is so low that the pulsation level only decays slowly. This is especially highlighted on the green curve during the suction phase by placing an envelope curve over the peaks of the pressure amplitude. After retrofitting the PRS to the pump, pressure measurements were taken at the same pump speed. The result of this measurement is shown in the bottom graph. As can be seen, all pressure pulsations are significantly reduced during both the suction and discharge phases. The energy dissipation becomes particularly clear when an enveloping curve is drawn around the pressure peaks during the suction phase. After only a few amplitudes, the pulsation comes to a complete stop, so that at the end of the suction phase the flow is absolutely calm before the compression phase follows.

Also, in the discharge phase a very clear and rapid decrease of the pressure pulsation can be seen. The sudden opening of an outlet valve of an adjacent diaphragm chamber results in a further pulsation stimulation in the middle of the discharge stroke, which is why the pressure curves are not so evenly calmed in the discharge phase. However, due to the effect of the PRS, all pressure curves are almost stabilised again at the end of the discharge phase.

Another point in comparing the two diagrams shows that the use of the PRS increases the minimum pressures in the diaphragm chamber during the suction phase. As can be seen from the green curve, the minimum pressure is about 1 bar for a short time when the pump is operated without PRS. By using the PRS, this minimum pressure is increased to over 2 bar if the pump is operated under otherwise identical boundary conditions. This pressure increase may sound very marginal at first, but it is of considerable significance when viewed under cavitation conditions. Cavitation always occurs when the pressure falls below the vapour pressure of the medium. However, this can be prevented if the pressure is only slightly raised above the vapour pressure, so a pressure increase of 1 bar can be very significant, as can be shown by NPSH calculations. Cavitation in particular impairs the operation of the pump and leads to increased wear, which is why the use of PRS is also recommended under these aspects. This effect is no coincidence and has been proven under other conditions and by simulation, as will be shown and explained later.

\subsection{Functionality of PRS}

The PRS is designed to effectively eliminate the high-frequency pulsations during the suction phase of the pump as well as during the discharge phase. Figure 5 shows how to attach the system to a triplex feed pump. The dampener units are each connected to the oil chamber of the triplex pump. Each PRS unit consists of a pressure-resistant housing, which is connected to the pump by means of a pressure line. The dampers can be activated or shut off via ball valves from the pump. To ensure that the oil can be absorbed at all, the PRS unit is pre-charged with nitrogen at a pressure matching the individual suction and discharge pressure of the pump.

The dampers work in such a way that a pressure pulsation in the diaphragm chamber of the pump is also transmitted to the oil volume. In the event of a brief pressure increase due to this pulsation, oil is displaced from the diaphragm chamber into the PRS unit. Like a shock absorber, this oil flow is guided through a throttle orifice. When passing the throttle orifice, part of the flow energy, and thus part of the pulsation energy, is converted to heat. During a brief pressure reduction, the absorbed oil is released by the PRS unit and passed through the orifice plate, which also dissipates energy. 


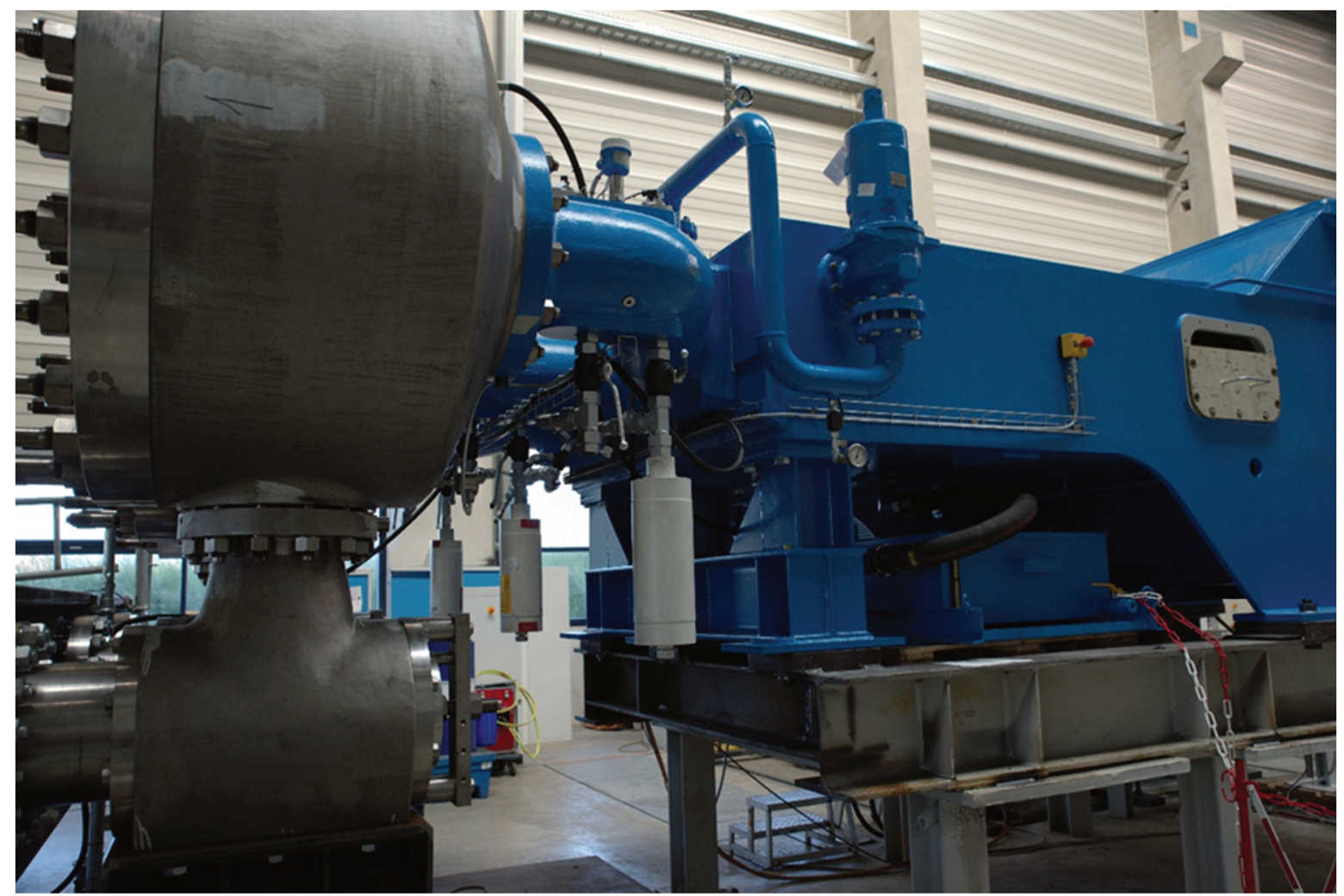

Figure 5 Installation of the PRS units to a positive displacement pump

\subsection{PRS in positive displacement pumps}

Due to the long distance between both capacitances of these pumps, positive displacement pumps are particularly susceptible to pulsations, as the columns of liquid accelerated by the piston movement in the long cooling line have a high kinetic energy. This increased pressure pulsation is also accompanied by higher mechanical stress in adjacent components, such as pipelines, fixtures in pipelines, diaphragm housings and the air tanks. Resonances, which can be excited by the pulsation frequencies, can excessively increase this mechanical stress. However, pressure peaks within the pump can also have a negative effect on the service life of the internal pump components (e.g. diaphragm and valves) and the pressure pulsations are reflected in the entire drive train of the pump.

Another aspect of positive displacement pumps is their tendency to cavitation. In order to prevent cavitation, the net pressure suction head required (NPSHr) must be maintained at all times. Temporary and repeated falling below that NPSHr is a risk for cavitation.

In the following we will explain how the retrofitting of PRS has a positive effect on these aspects in the pumps.

Analogous to Figure 3, the same measurements with PRS were carried out during a test of the same positive displacement pump. The oil chamber of the diaphragm housings of the triplex pump were equipped with PRS units. Due to the characteristics of the test field, the suction pressure was limited to approximately 6.5 bar. However, during the discharge phase the pressure was set to 57 bar and thus similar to the typical pressure of a positive displacement pump. Figure 6 shows the result of this measurement. 


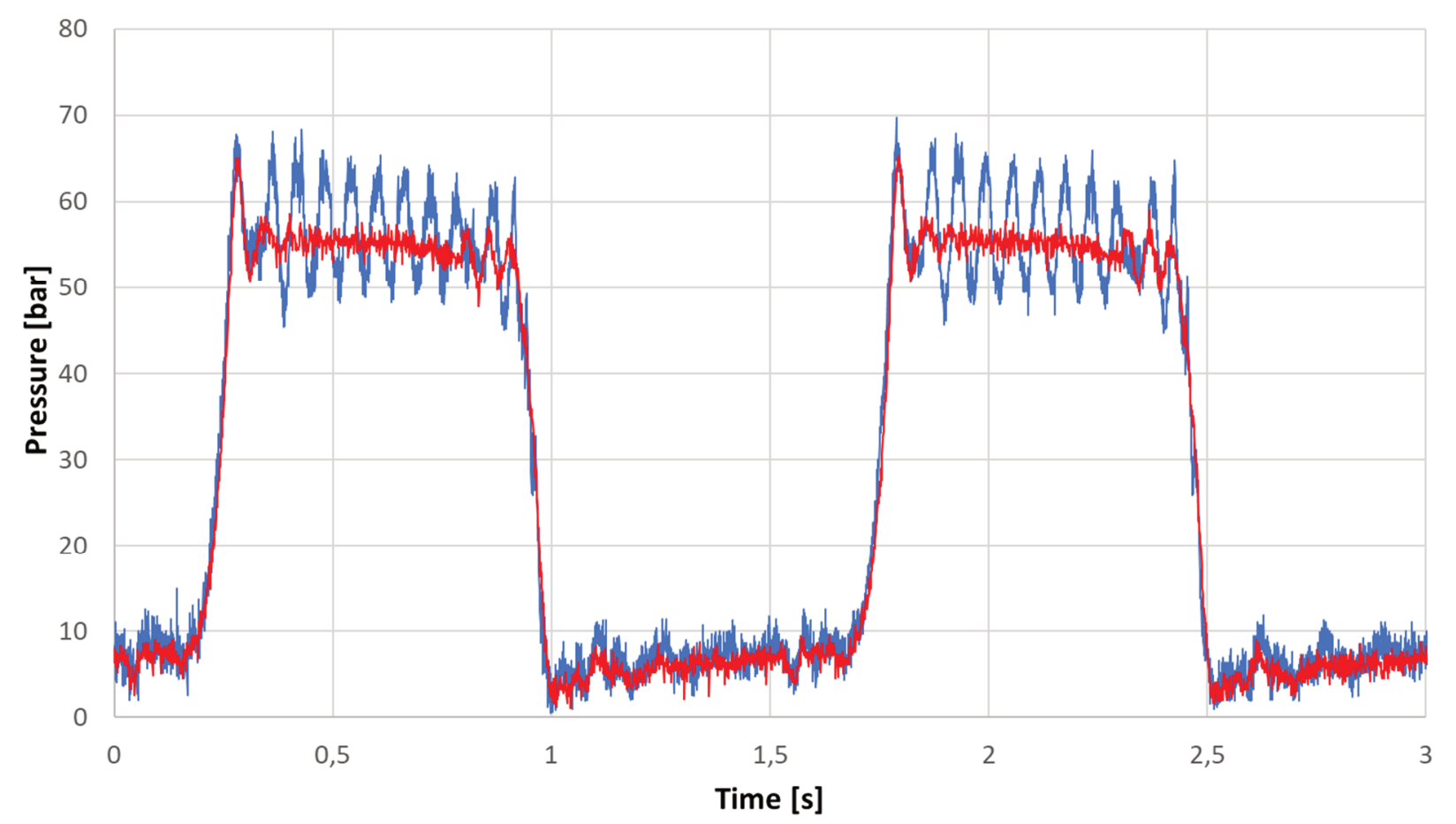

Figure 6 Pressure measurement in positive displacement pump - red with PRS, blue without PRS

The measurement shows in red the pressure curve in the diaphragm housing when the PRS unit is activated. In order to show the differences to the measurement without PRS, the measurement at identical speed and pressure from Figure 3 was highlighted in blue. The red curve indicates during the discharge phase of the pump that the high pulsations are eliminated very effectively and very quickly by the PRS. The energy dissipation here is so high that after an initial pressure peak the oscillation almost comes to a standstill. At the end of the discharge phase there is still a slight pressure excitation, since, as already described, the outlet valve of an adjacent diaphragm chamber opens at this point and feeds into the common pressure manifold.

While the piston chamber is in suction mode, pulsations are also visible in the blue curve, although with a lower amplitude. These are also damped by the PRS unit, as the red curve in the diagram shows.

\subsection{Simulation of PRS}

Since the pressure shown in Figure 4 does not reflect the exact pressure in the pumps during operation, simulations were carried out to simulate the behaviour of the positive displacement pump with PRS under real operating conditions. For this purpose, the simulation software SimulationX was used, with which pump systems have been successfully simulated at MHWirth for years and continuously adjusted by field measurement and experience at several applications and pump units. SimulationX is a multiphysical system simulation, and components and parts from up to large systems can be modelled from different technical disciplines, such as mechanical engineering, hydraulics, electrical engineering and many other disciplines.

To give an impression of the simulation model of the PD pump, a section of the simulation model of this pump is shown in Figure 7. All essential aspects, such as hydraulic capacitances and inductances, flow resistances or the thermodynamic behaviour of the gases are represented in this model and based on the real component dimensions. The behaviour of the valves including a flow and resistance force model is also considered in the simulation. The medium to be pumped can be simulated by its physical properties such as viscosity, density, compressibility and vapour pressure. 


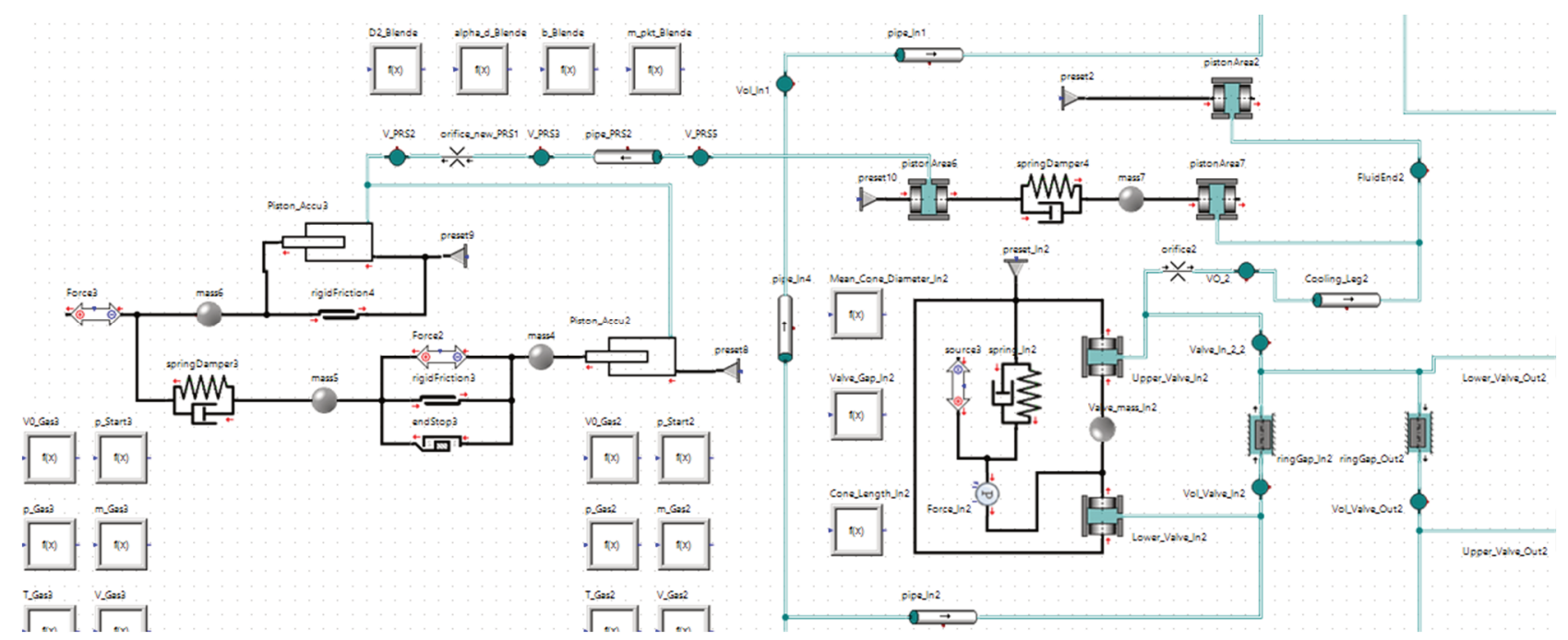

Figure 7 Extract from the simulation model of the positive displacement pump

As a result of this simulation, state variables such as pressures, flow rates, temperatures and forces can be recorded and visualised in the time domain at each node of the model. In Figure 8 the result of the simulation can be seen by means of the pressures in the diaphragm chamber. The speed of the pump is $40 \mathrm{rpm}$, the suction pressure is set to 18 bar and the outlet pressure to 57 bar. In the discharge phase, the pulsation height and the frequencies without PRS (blue curve) correspond quite well with the measurements mentioned above. This shows that the simulation is very close to reality.

The red curve indicates the pressure behaviour when using PRS. Here too, the course of the measured curve in Figure 6 corresponds very well with that of the simulated curve. Therefore, it can be said that the damping behaviour in the simulated PRS unit has a high agreement with the measured PRS unit.

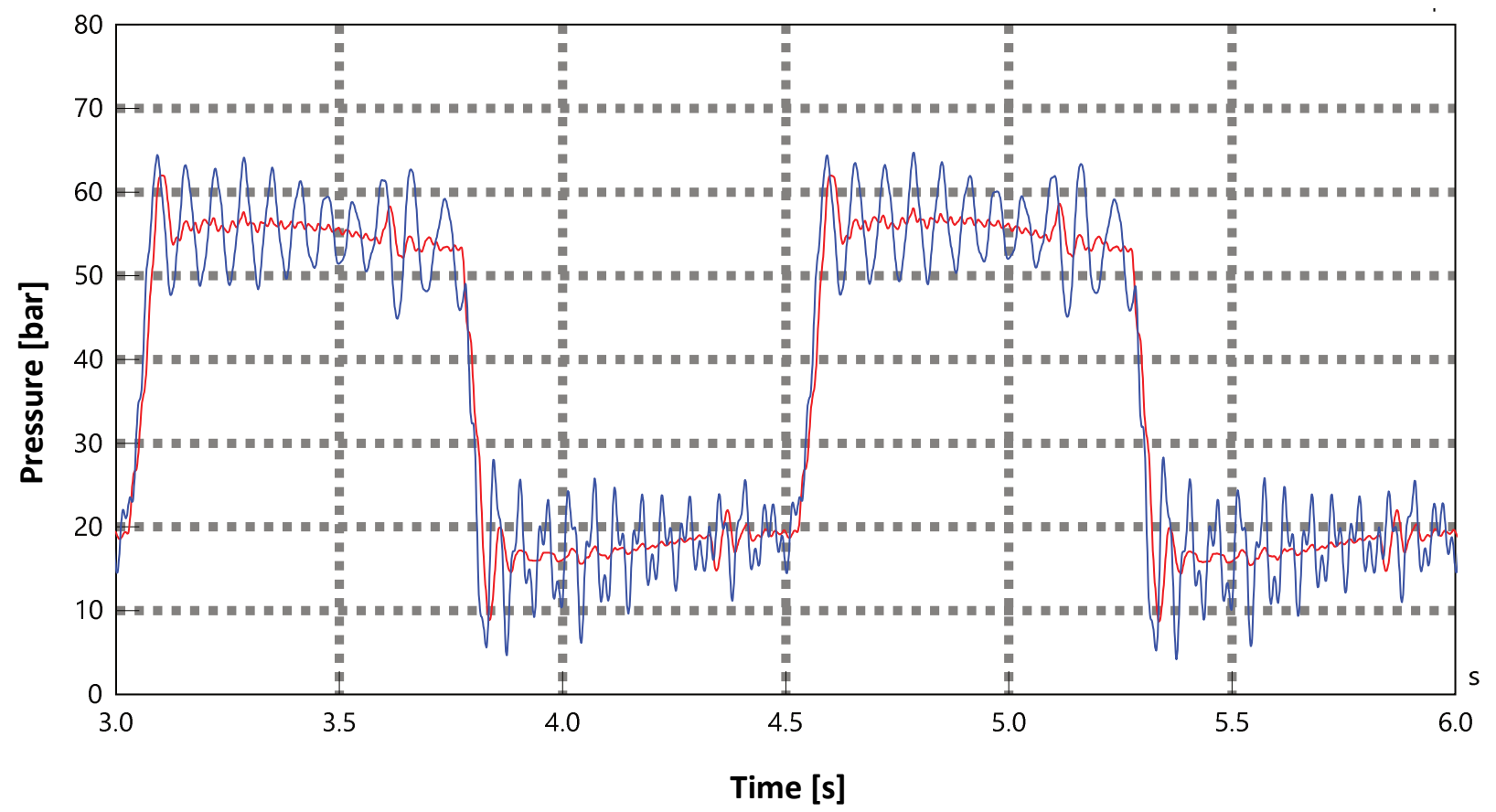

Figure 8 Simulation of chamber pressure with PRS (red) and without PRS (blue)

In the simulation without PRS, clear pulsations also occur during the suction phase. These are also quickly eliminated by the PRS, as the red curve shows. Another aspect during the suction phase relates to cavitation. The use of PRS makes it possible to reduce the pulsation to such an extent that even the first peak that occurs 
after the suction valve is opened has a lower amplitude. As a result, the absolute pressure minimum is raised during the suction phase, which reduces the tendency to temporary cavitation followed by water hammer effects. Compared to the behaviour without PRS, the minimum pressure in the diaphragm chamber can be raised by approximately 4 bar. The further flattening of the pressure curve also prevents the repeated approach to this low-pressure range.

\section{$4 \quad$ Risk assessment}

In addition to the shown advantages that a PRS offers on the operation of the pump, the aspects that may have a negative effect on the performance of the pump will also be examined.

One of these aspects refers to the volumetric efficiency of the pump. Since during a pump cycle a part of the oil volume displaced by the pump piston does not enter the diaphragm chamber but streams into the PRS units, this part is no longer available to perform compression or pumping work on the slurry. For this reason, the volumetric efficiency of the pump will decrease. Since the reduction of the flow rate of the pump by this effect is relatively small and could barely be measured, a simulation of a pump with and without PRS was compared. According to this simulation, the PRS reduces the flow rate delivered by the pump by $1.2 \%$, i.e. the volumetric efficiency is reduced by this percentage when the pump is operated under otherwise exact conditions. This value also corresponds mathematically to the volume of oil delivered and received by the PRS per cycle.

However, it should be noted that this lower flow rate can be compensated very easily by operating the pump at a speed increased by $1.2 \%$. This should be possible without any problems due to the almost non-existent pulsations. MHWirth considers this effect already in the design phase of the pump, should PRS be considered in a future project.

With the help of simulation, it is also possible to capture sizes that are otherwise difficult to measure because they are difficult to determine. One of these measured variables is the energy that is converted into heat at the throttle elements. Since this energy leads to an increase in the oil temperature, this aspect is of course of particular importance to ensure operational safety. The power that is converted into heat at the orifice plate depends strongly on the operating conditions of the pump. Particularly, the differential pressure between the inlet and outlet side of the pump, and the speed of the pump have a strong influence on this heat input. For the cycle considered in Figure 6, the heat dissipation per PRS unit is $820 \mathrm{~W}$. This heat is completely absorbed by the oil. This additional heat input appears relatively small compared to the power converted by the pump and the associated power losses. It should not lead to an excessive temperature increase due to the large oil volume of a fluid end of approximately $200 \mathrm{I}$, which is surrounded by a steel housing of several tonnes. These observations were also made in the field with the installations that have been running since February 2019. These pumps are located outdoor in a desert environment and are operated under extremely hot climatic conditions without overheating the PRS units, although they are running without additional external cooling.

From a maintenance point of view, it is required to pre-charge the accumulators to the average discharge pressure. The nitrogen pressure for damping during the suction phases is automatically adjusted by the integrated valve technology. As with a conventional bladder accumulator, the existing pre-charge pressure should be checked from time to time. If leakage occur, the gas charge should be refilled accordingly. However, the system is very fault-tolerant in this respect, as pulsation damping is still present, even if the pre-charge pressure has been reduced by $20 \%$ due to gas leakage. The seals used in the PRS are designed as spare parts and can be replaced if necessary or during a routine inspection of the pump. Apart from that, there is no regular maintenance activity required.

An additional system installed to the pump may bear the risk of an unforeseen failure which is then affecting the operation. The new PRS is installed with a shut off valve so that it can be set to active or inactive very quickly. Should there be any failure with the PRS it can simply be decoupled from the system, allowing the pump to operate as usual. 


\section{Conclusion}

In this paper it is shown that high-frequency pulsations are always present in the described pumping systems. However, the size and frequency may vary due to different pump designs and operational conditions of the pumps. Referring to the calculations introduced in Section 2, the hydraulic oscillation would change with different values for inductance or capacitance. Also, due to natural frequency and resonance effects, the negative impact of pressure pulsations can be amplified.

Site tests have shown that the risk for fatigue failures, overstress of the pump power end, and the risk for cavitation during each stroke can be reduced tremendously, thus increasing the lifetime of components and reducing operational costs of the process.

To eliminate these high-frequency pulsations, a new type of damping system was developed; the so-called pulsation reduction system or PRS. With the help of the PRS, the high-frequency pressure pulsations are converted into heat and extinguished.

The low-frequency pulsations induced by the non-uniform volumetric flow of the piston movement must still be compensated by conventional damping elements, such as air vessels on the suction and discharge side. The PRS system provides a very useful addition to the conventional damping methods and can easily be retrofitted to pumps of any brand or model which are already in operation.

By using the PRS, the high-frequency pulsations on both the suction and the discharge side of the pump are almost completely eliminated which leads to:

- Reduced resonances and mechanical vibrations in the pump and the surrounding components and pipework.

- Reduced force and stress peaks in the drivetrain of the pump.

- Limited cavitation effects.

- Limited water hammer effects.

- Reduced wear and tear of all pressure loaded parts.

- Increased lifetime of consumables, like valves and diaphragms.

- Increased pump availability.

Therefore, it is recommended to install the unique PRS, which is invented and patented by MHWirth, to every positive displacement slurry pump working under heavy duty conditions of $>150 \mathrm{~m}^{3} / \mathrm{h}$ and $>40$ bar.

For the future, this new technology is expected to offer another major advantage: since the pulsation height of conventional piston pumps increases strongly with the speed of rotation, pulsation is one of the main reasons for limiting the speed of these pumps. Here, the PRS technology opens up new possibilities to increase the speed of the pump and thus to increase the pumping capacity within the same pump size.

\section{Acknowledgement}

We thank the following companies for their assistance with the collection of the data:

- BHP, https://www.bhp.com, Olympic Dam Project, operating four MHWirth DPM1250-22, https://www.bhp.com/our-businesses/minerals-australia/olympic-dam

- OZ Minerals, https://www.ozminerals.com, Carrapateena Project, operating one MHWirth TPM2200-20 https://www.ozminerals.com/operations/carrapateena-project 


\section{References}

Jolly, P \& Cocks, A 2017, 'Application of positive displacement pumps in HPAL circuits', Proceedings of ALTA 2017 Nickel-Cobalt-Copper Sessions, ALTA Metallurgical Services Publications, Perth, pp. 395-409.

Murrenhoff, H 1998, Grundlagen der Fluidtechnik, Teil 1: Hydraulik (Fundamentals of Fluid Technology, Part 1: Hydraulics), Verlag Mainz, Aachen.

Schade, O 2009, 'Kavitation in Oszillierenden Verdraengerpumpen (Cavitation in oscillating displacement pumps)', in E Schlücker (ed.), Schriftenreihe des Lehrstuhls für Prozessmaschinen und Anlagentechnik - Serie 8, Shaker Verlag, Aachen.

Vlot, E 2018, 'Dynamics of piston diaphragm pumps in autoclave feed applications', Proceedings of ALTA 2018 Nickel-Cobalt-Copper Sessions, ALTA Metallurgical Services Publications, Perth, pp. 1-10. 